\title{
Impact of the seven-valent pneumococcal conjugate vaccination (PCV7) programme on childhood hospital admissions for bacterial pneumonia and empyema in England: national time-trends study, 1997-2008
}

\author{
Elizabeth Koshy, ${ }^{1}$ Joanna Murray, ${ }^{1}$ Alex Bottle, ${ }^{1}$ Mike Sharland, ${ }^{2}$ Sonia Saxena ${ }^{1}$
}

\begin{abstract}
- A supplementary appendix is published online only. To view this file please visit the journal online (http://thorax.bmj.com).

'Department of Primary Care and Public Health, Imperial College London, UK

${ }^{2}$ Paediatric Infectious Diseases Unit, St George's Hospital NHS Trust, London, UK
\end{abstract}

\section{Correspondence to}

Dr Sonia Saxena, Department of Primary Care and Public Health, Room 332, Reynolds Building, Imperial College London, Charing Cross Campus, London W6 8RP, UK:

s.saxena@imperial.ac.uk

Received 24 February 2010 Accepted 12 May 2010

\section{ABSTRACT}

Background Childhood bacterial pneumonia and empyema rates have reportedly increased in recent years in Europe. In September 2006 the seven-valent pneumococcal conjugate vaccination (PCV7) was introduced to the childhood national immunisation programme in England following a successful PCV7 campaign in the USA. The aim of this study was to report national time trends in hospital admissions for childhood bacterial pneumonia and empyema in England before and after the introduction of PCV7.

Methods A population-based time-trend analysis of Hospital Episode Statistics data of children aged $<15$ years admitted to all NHS hospitals in England, with a primary diagnosis of bacterial pneumonia and empyema from 1997 to 2008 was performed. Annual crude and age-sex standardised hospital admission rates for bacterial pneumonia and empyema were calculated. Results Admission rates for bacterial pneumonia and empyema increased from 1997 to 2006, then declined to 2008. Bacterial pneumonia rates decreased to 1079 (95\% Cl 1059 to 1099) per million children and empyema rates decreased to 14 (95\% $\mathrm{Cl} 11$ to 16) per million children. The RR for bacterial pneumonia admissions was 1.19 (95\% Cl 1.16 to 1.22) in 2006 compared with 2004 and 0.81 (95\% Cl 0.79 to 0.83 ) in 2008 compared with 2006. For empyema, the corresponding RRs were 1.77 (95\% Cl 1.38 to 2.28) in 2006 compared with 2004 and $0.78(95 \% \mathrm{Cl} 0.62$ to 0.98$)$ in 2008 compared with 2006.

Conclusion Childhood bacterial pneumonia and empyema admission rates were increasing prior to 2006 and decreased by $19 \%$ and $22 \%$ respectively between 2006 and 2008, following the introduction of the PCV7 pneumococcal conjugate vaccination to the national childhood immunisation programme.

\section{BACKGROUND}

Acute respiratory tract infections (RTIs) are the most common cause of admissions to hospital for children of all ages and pose a significant burden of illness on families as well as on primary and secondary healthcare services. ${ }^{1}{ }^{2}$ Most childhood RTIs are self-limiting viral infections, but a small proportion develop into serious bacterial RTIs such as pneumonia and empyema (pyothorax) which are predominantly caused by Streptococcus pneumoniae. There was some evidence that falls in antibiotic prescribing since the mid-1990s ${ }^{3}{ }^{4}$ exposed vulnerable groups such as children and elderly people to a higher risk of morbidity and mortality from bacterial RTIs. ${ }^{4-6}$ Recent studies suggest that rates of childhood empyema are increasing in the $\mathrm{UK}^{7-10}$ and other developed countries. ${ }^{11-14}$ Adult bacterial pneumonia admission rates have continued to increase in England in recent years despite a targeted primary care pneumococcal vaccination programme for patients aged $>65$ years and some high-risk groups for pneumonia since $2003 .{ }^{15}$

In the USA a successful seven-valent pneumococcal conjugate vaccine (PCV7) infant immunisation programme was introduced in February 2000 and by 2004 a 39\% decrease in admission rates for pneumonia in children aged $<2$ years was observed. ${ }^{16}$ PCV7 offers protection against the most common serotypes which have accounted for most of the bacterial pneumonias in children. ${ }^{15} 17$ Empyema is caused by $S$ pneumoniae serotype 1 in up to half of the cases in the UK and USA. ${ }^{18} 19$ The serotypes covered by PCV7 are 4, 6B, 9V, 14, 18C, 19F and 23F. Notably, serotype 1 is not covered by this vaccine. In September 2006 PCV7 was introduced into the UK primary childhood immunisation programme administered at 2, 3 and 13 months of age, with a catch-up campaign for children up to 2 years. During 2007-8 the uptake of PCV7 among children in England was reported as 84\% by their first birthday (http://www.ic.nhs.uk/), a level high enough to contribute towards early herd immunity.

The UK Health Protection Agency (HPA) reported a marked reduction in the rate of cumulative increase of cases of childhood invasive pneumococcal disease (IPD) caused by the seven serotypes contained in PCV7 within the first year of the programme but, by the end of 2009, increases in non-PCV7 strains of childhood IPD had risen to match numbers reported before the introduction of the vaccine..$^{20}$

IPD is a serious bacterial infection defined by the isolation of $S$ pneumoniae from a normally sterile site. It includes bacterial pneumonia, meningitis, septicaemia and empyema and does not cover the full spectrum of clinical disease. The HPA only reports microbiologically-confirmed cases with no denominator data to provide rates. The population impact of this vaccine on childhood bacterial pneumonia and empyema in England has therefore not been evaluated. Despite this, the Department of Health has now switched from PCV7 to PCV13 (a vaccine active against 13 serotypes of $S$ pneumoniae) in April 2010, only 3 years after the national vaccination policy was rolled out. We conducted 
a national time-trends study of admission rates for childhood bacterial pneumonia and empyema over a time period spanning the PCV7 vaccination campaign.

\section{METHODS}

The Hospital Episode Statistics database (http://www.hesonline.org.uk) contains information on all episodes of NHS (public) hospital care in England. The data are recorded as finished consultant episodes, which are defined as the time period during which an admitted patient is under the care of a particular hospital consultant. The main reason for admission ("primary diagnosis') is coded using the International Classification of Diseases version 10 (ICD-10) codes, ${ }^{21}$ with up to 13 secondary diagnoses. We used the primary diagnosis field to capture admissions for common community-acquired RTIs as opposed to hospital-acquired RTIs. We excluded RTI admissions where there was a comorbid diagnosis of cystic fibrosis (CF) in one of the subsidiary diagnosis fields, as this disease can contribute to a large number of recurrent hospital-acquired RTI admissions. We calculated annual emergency hospital admission rates in children aged <15 years between calendar years 1997 and 2008 using ICD-10 codes for viral RTIs, bacterial pneumonia and empyema (see table AI in online appendix). Studying viral RTI trends enabled us to determine whether trends in childhood bacterial pneumonia and empyema were independent or secondary to changes in admissions for all paediatric respiratory infections. We calculated all-cause emergency admission rates for comparison with RTI admission rates. The rates for boys and girls were calculated separately and in combination in four distinct age categories (<1 year, 1-4 years, 5-9 years, $10-14$ years) reflecting key stages of childhood development for comparison with previous studies. ${ }^{8} 10$

Mid year population estimates for year, age and sex obtained from the Office of National Statistics (http://www.statistics. gov.uk) were used to calculate age- and sex-specific and agestandardised admission rates per million children and associated $95 \%$ CIs. To compare admission rates before and after the introduction of the vaccine in September 2006, we calculated the age-sex standardised RR of admission in 2006 compared with 2004 and in 2008 compared with 2006. STATA/MP Version 10 software (STATA, College Station, Texas, USA) was used for all statistical analyses.

\section{RESULTS}

Between 1997 and 2008 there were 7295551 emergency hospital admissions for children aged $<15$ years. Bacterial pneumonia and empyema accounted for $2 \%$ (138197) and viral RTIs accounted for $12 \%$ (893077) of these admissions. We excluded 6656 RTI admissions with co-existing CF during the 12-year study period.

Bacterial pneumonia admission rates increased by $31 \%$ from 1022 (95\% CI 1003 to 1042) per million children in 1997 to 1340 (95\% CI 1317 to 1362 ) in 2005. By 2008 the rate had fallen by $19 \%$ to 1079 (95\% CI 1059 to 1099) per million children (table 1, figure 1). Comparing admission rates 2 years before and after the introduction of PCV7 in 2006 gave a RR of admission for bacterial pneumonia of 1.19 (95\% CI 1.16 to 1.22$)$ in 2006 compared with 2004 and 0.81 (95\% CI 0.79 to 0.83 ) in 2008 compared with 2006.

The number of admissions for empyema increased from 50 in 1997 to 140 in 2008 (table 1). Age-sex standardised admission rates for empyema increased from 5 (95\% CI 3 to 6 ) per million children in 1997 to 18 (95\% CI 15 to 20 ) in 2006 . The rate then declined by $22 \%$ to 14 ( $95 \%$ CI 11 to 16 ) per million children in 2008. Comparing admission rates 2 years before and after PCV7 gave a RR of empyema admission of 1.77 (95\% CI 1.38 to 2.28) in 2006 compared with 2004 and 0.78 (95\% CI 0.62 to 0.98 ) in 2008 compared with 2006. This consistent pattern of sharp increases before and falls after the introduction of PCV7 for bacterial pneumonia and empyema was seen against a more steady background trend of increasing emergency admissions for viral RTI (table 1). The RR for viral RTI admissions was 1.04 (95\% CI 1.03 to 1.05 ) in 2006 compared with 2004 and 1.09 (95\% CI 1.08 to 1.10) in 2008 compared with 2006. Similarly, the emergency age-sex standardised RR for all-cause admissions was 1.08 (95\% CI 1.08 to 1.09 ) in 2006 compared with 2004 and was 1.04 (95\% CI 1.03 to 1.04) in 2008 compared with 2006.

The highest bacterial pneumonia admission rates in all study years were seen in younger age bands (children aged $<1$ year and 1-4 years). A decrease in pneumonia admission rates from 2006 to 2008 occurred across all age groups (see table AII in online appendix). Boys had higher bacterial pneumonia admission rates than girls in all age categories throughout the 12-year period studied (figure 2 and table AII in online appendix). The highest empyema admission rates were predominantly in children aged

Table 1 Annual trends in hospital admissions for bacterial pneumonia, empyema, viral respiratory tract infections (RTIs) and all-cause admissions

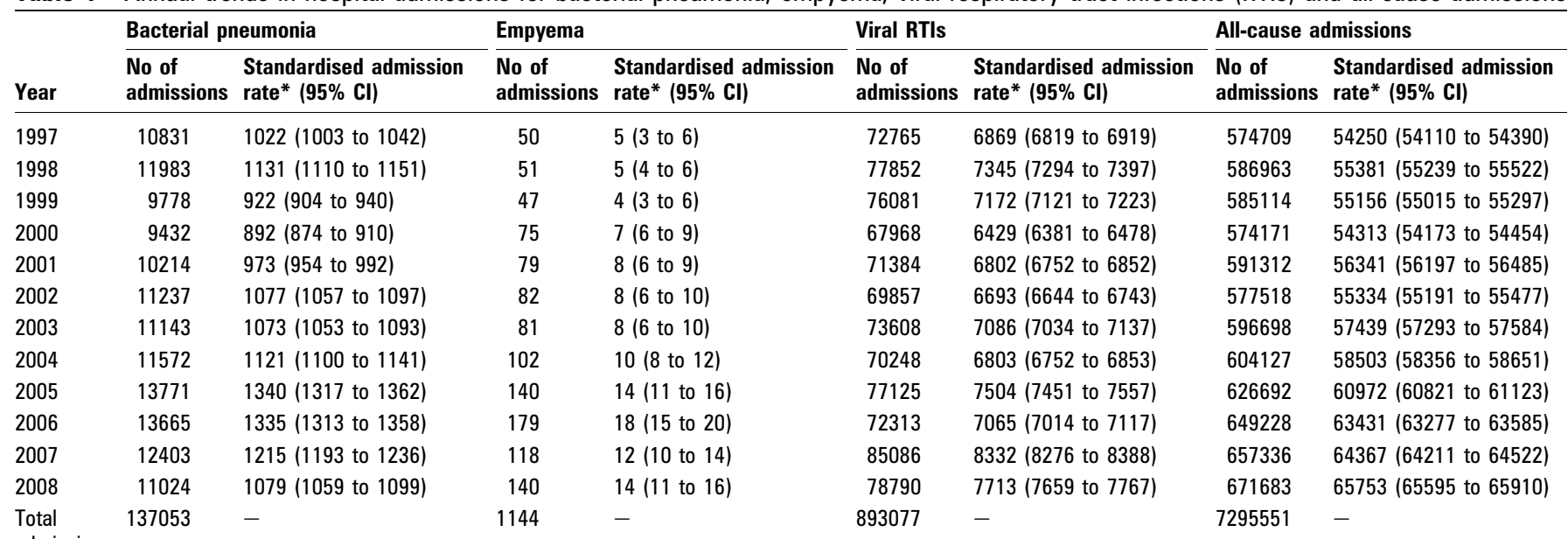

admissions

*Admission rates standardised by age and sex per million children. Mid year population estimates from the Office of National Statistics (ONS) were used to calculate rates. 95\% Cls for all rates shown in brackets. 


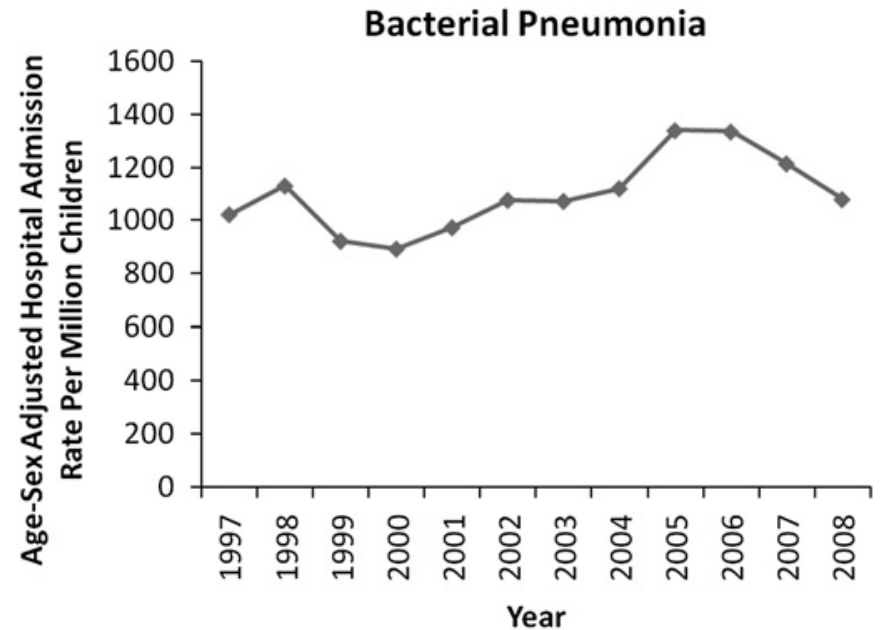

Figure 1 Annual hospital admission rates for childhood bacterial pneumonia (age $<15$ years).

$<5$ years. The largest increase in empyema rates between 1997 and 2008 was in the age group 1-4 years for both boys and girls (see table AIII in online appendix).

\section{DISCUSSION}

Bacterial pneumonia and empyema rates in children aged $<15$ years admitted to hospitals in England increased from 1997 to 2006 , then declined by $19 \%$ and $22 \%$ respectively in the 2 years following the introduction of the childhood PCV7 pneumococcal vaccination programme in the UK in September 2006.

\section{Strengths and limitations}

To our knowledge, this is the first study to report trends in bacterial pneumonia and empyema admission rates in children since the introduction of PCV7 in England. Its strengths lie in its large nationally representative population-based sample providing information about the clinical impact of PCV7 on the incidence of bacterial pneumonia and empyema, which is an important adjunct to laboratory-confirmed case reports from national surveillance and regional studies.
However, our study has a number of important limitations. The accuracy of HES depends on clinical diagnoses recorded in patients' medical records. In recent years the quality of coding has improved and HES data have been used extensively for research. ${ }^{22}$ Since the coding is based on clinical and microbiological information recorded by clinicians upon discharge, the distinction between viral and bacterial RTI diagnoses is potentially subject to misclassification bias.

\section{Findings related to other studies}

Year-on-year rises in admission rates for childhood bacterial pneumonia prior to 2006 in our study are comparable to those reported in Scotland ${ }^{10}$ and the Netherlands. This lends support to a true increase in incidence rather than improvements in HES coding since its inception. ${ }^{23}$ Our findings that all-cause emergency admission rates in children increased throughout the period studied are consistent with a wider trend of steadily rising unplanned hospital admission rates in children in England, ${ }^{24}$ but are modest in comparison with the sharper increases in bacterial pneumonia. In contrast to the continuing rise in all-cause hospital admissions, bacterial pneumonia and empyema admission rates have decreased since 2006. There was a similar steady background trend of increasing emergency admissions for viral RTI. Childhood bacterial pneumonia and empyema trends therefore seem to be independent of changes in admissions for other paediatric respiratory infections.

One possible (but unlikely) explanation for rises in bacterial pneumonia admission rates is increasing antibiotic-resistant pneumococcal infections. However, HPA reports suggest that the numbers of penicillin-resistant isolates from pneumonia cases were relatively stable prior to $2006 .^{20} \mathrm{~A}$ more plausible explanation is that patterns of bacterial pneumonia mirror antibiotic prescribing rates in primary care, which fell dramatically in the late 1990s and were reportedly associated with increased hospital admissions for RTIs in adults. ${ }^{4}$ Since then, primary care antibiotic prescribing rates appear to be increasing again. ${ }^{25}$ The total antibiotic prescribing rate for children in UK primary care decreased by $24 \%$ between 1996 and 2000, was relatively stable between 2000 and 2003, and increased by $10 \%$ between 2003 and $2006 .^{25}$ Trends in antibiotic prescribing rates closely mirror
Figure 2 Annual hospital admission rates for bacterial pneumonia by age group: (A) $<1$ year; (B) $1-4$ years; (C) 5-9 years; and (D) 10-14 years.
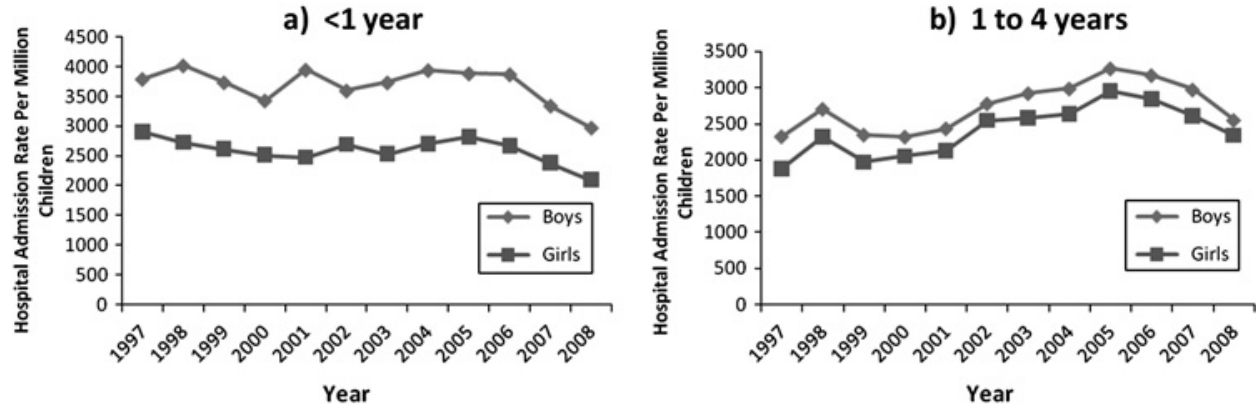

c) 5 to 9 years
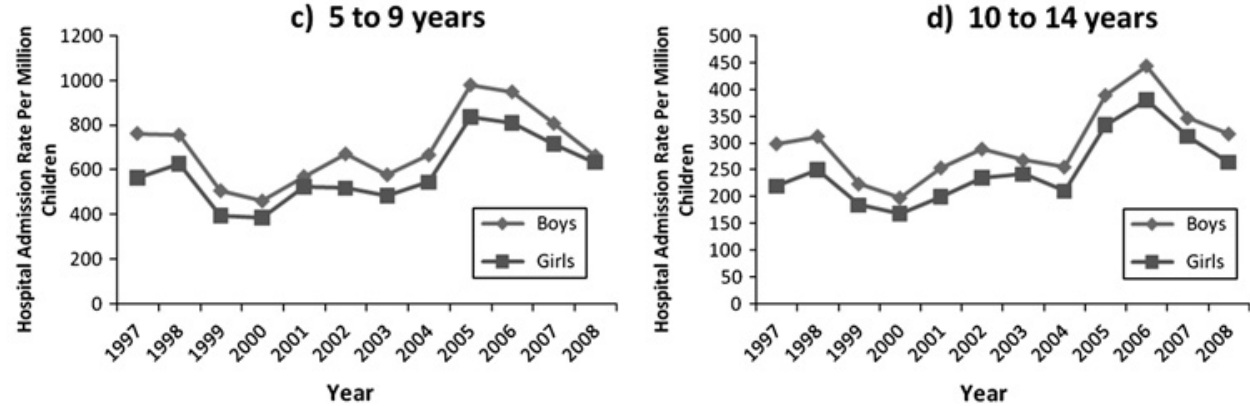
consultation rates for childhood RTI in primary care, ${ }^{25}$ hospital admission rates and mortality related to respiratory infection for all ages. ${ }^{26}$ We therefore contend that the most likely explanation for our observed trends in bacterial pneumonia admission rates is a growing incidence of childhood RTI in the community prior to 2006. We found no studies which enabled us to compare the pattern of antibiotic prescribing with decreasing admission rates for bacterial pneumonia and empyema since 2006.

Children in the youngest age groups ( $<1$ year and $1-4$ years), among whom the incidence of IPD is known to be high, ${ }^{15}$ had the highest admission rates for bacterial pneumonia in our study. Although PCV7 was targeted at children aged $<2$ years, ${ }^{15}$ our study shows evidence of herd immunity arising among older children since we found decreasing bacterial pneumonia admissions from 2006 to 2008 among all age groups.

Empyema admission rates increased between 1997 and 2006 (from 5 to 18 per million children) and subsequently mirrored the falls in bacterial pneumonia following PCV7. Several countries have reported increasing childhood empyema rates, but our findings are lower than those reported in other UK studies ${ }^{8} 10$ and countries including Spain and Israel. ${ }^{12}{ }^{13}$ A Scottish study by Roxburgh et al found that admissions increased after 1998 from $<10$ per million children per annum to reach a peak of 37 per million in $2005 .{ }^{10}$ Gupta et $a l^{8}$ reported an increase in the rate from 14 cases per million in 1995/6 to 26 cases per million in $2002 / 3$. We used more specific ICD codes for empyema than this study, which could explain the lower number of cases we identified. Since the numerator is based on a small number of empyema admissions, our estimates are less robust than those for bacterial pneumonia. However, the low incidence of empyema cases and the increasing trend that we observed are consistent with these studies and so are likely to be real. Improvements in detection rates with increased use of imaging techniques or interventional procedures might explain the initial increases in empyema rates seen. Alternatively, the increasing empyema trend could be due to changes and variations in the natural ecology of the causative organisms. However, Saglani et al found that the majority of organisms detected in the pleural fluid of patients with childhood empyema were $S$ pneumoniae, $S$ pyogenes, Staphylococcus aureus and anaerobic bacteria, and so concluded that the recently described increases in childhood empyema were not ascribable to unusual pathogens. ${ }^{27}$ Similarly, attributing the rise to increased penicillin-resistant $S$ pneumoniae has been refuted by studies which identified only fully sensitive $S$ pneumoniae organisms. ${ }^{18}{ }^{27}$ Individual case reviews of empyema admissions through a disease registry could provide more information about this serious bacterial complication.

The pattern of a rise followed by a fall in childhood bacterial pneumonia and empyema admission rates before and after the introduction of PCV7 may mean that vaccine-sensitive pneumococcal strains could account for up to one-fifth of cases of bacterial pneumonia and empyema. We observed a 19\% decline in pneumonia rates following the introduction of PCV7, which is half the reduction of $39 \%$ reported in the USA. ${ }^{16}$ However, this US study looked at rates 4 years after PCV7 whereas our study shows rates 2 years after PCV7, so further falls may have occurred given the longer lead time. Empyema is almost always a complication of pneumonia, so the reduction in PCV7 serotype bacterial pneumonia rates may have contributed to the observed decline in empyema rates after PCV7. S pneumoniae serotype 1 is the causative organism for around one-half of empyema cases and is not covered by PCV7. ${ }^{28}$ The fall in childhood empyema admission rates after PCV7 therefore suggests that other PCV7 strains might also be important causal organisms in empyema.
Potential benefits of pneumococcal vaccination extend beyond the target population by reducing carriage and transmission of vaccine-serotype pneumococci and could, as reported in the USA, reduce bacterial pneumonia in unvaccinated children aged $5-17$ years $^{29}$ and adult cohorts. ${ }^{29} 30$

Has there been true serotype replacement or are the findings of increasing invasive pneumococcal disease due to ascertainment bias secondary to an enhanced surveillance programme? The HPA reports from 2003 onwards suggest that there has been some clear serotype replacement. The cumulative weekly number of IPD cases due to any of the PCV7 serotypes in children aged $<5$ years and $\geq 5$ years has decreased dramatically since the introduction of PCV7. ${ }^{17}$ However, this has not been the situation with non-PCV7 serotypes where IPD numbers are now higher than before the PCV7 programme in both age groups. ${ }^{17}$ Experience in the USA following the introduction of PCV7 in 2000 has also demonstrated shifts in serotype prevalence after PCV7. ${ }^{19} 2931$ The replacement serotypes among IPD cases in England are not identical to those observed in the USA. In England serotypes 7F, 19A and 22F have emerged as common non-PCV7 serotypes. ${ }^{17}$ In the USA, in addition to these three serotypes, non-PCV7 serotypes 3, 12F, 15B/C, 33F and $38^{29} 32$ have been frequently isolated. Serotype replacement for IPD cases in England was observed within a year of introducing PCV7 and may have occurred faster than in the USA. ${ }^{20} 29$ Possible reasons for this could stem from England having a three-dose schedule for PCV7 whereas the USA has a four-dose schedule. ${ }^{33}$ Substantially higher broad-spectrum antibiotic prescribing rates in the USA ${ }^{34}$ may also account for lower serotype replacement, possibly due to the impact on nasopharyngeal colonisation.

\section{Implications and future research}

A childhood pneumococcal vaccine with broader coverage containing 13 serotypes (PCV13) has now replaced PCV7 from Spring 2010. The HPA has suggested that up to an extra $50 \%$ of the remaining IPD in England and Wales is preventable with PCV13. ${ }^{20}$ However, the HPA has also reported that the percentage of IPD cases in children aged $<5$ years caused by strains covered by PCV13 decreased from $92 \%$ in 2005/6 to $74 \%$ in $2007 / 8$. In children aged $\geq 5$ years the percentage of IPD cases caused by PCV13 serotypes was similar in 2005/6 and 2007/8, with only $67 \%$ of cases covered in $2007 / 8$. This has important implications for herd immunity. It also raises the question whether the introduction of PCV13 will lead to the emergence of new strains with yet more serotype replacement. It is difficult to predict PCV outcomes and future serotype replacement. There are over $90 S$ pneumoniae serotypes. PCV13 only contains six additional serotypes (1, 3, 5, 6A, 7F and 19A) compared with PCV7, and notably not $22 \mathrm{~F}$ for which there has already been considerable serotype replacement in England and Wales. ${ }^{17}$ It is therefore likely that further serotype replacement will continue. Long-term clinical phenotype, epidemiological and microbiological monitoring, ideally linked to antibiotic prescribing, is required to follow these trends. Additionally, more research is needed to study the factors which influence serotype replacement and the rate at which this occurs, including the role of primary care antibiotic prescribing.

The potential success of any vaccination programme depends on maintaining high uptake rates. General practitioners will need to explain proposed changes to the vaccination schedule to parents to reassure them, in clinically meaningful terms, that the proposed changes are likely to benefit their child. Our study highlights the fact that vaccinating the target population with PCV7 may have led to a $19 \%$ reduction in bacterial pneumonia and $22 \%$ reduction in empyema hospital admission rates within 
the first 2 years. Smaller decreases among unvaccinated older children suggest there may be herd immunity. This information will be useful to doctors advising parents on the benefits of vaccinating their child against $S$ pneumoniae. It may be reassuring to parents to know that, despite recent concerns of serotype replacement, PCV7 appears to have had a substantial impact on reducing the burden of illness from pneumonia and empyema.

Funding This study represents independent research commissioned by the National Institute for Health Research Service Delivery and Organisation programme. The interpretation and conclusions contained in this study are those of the authors. EK is funded by a National Institute for Health Research doctoral fellowship. SS is funded by a National Institute for Health Research postdoctoral fellowship. AB and the Dr Foster Unit at Imperial are funded via a research grant from Dr Foster Intelligence, an independent healthcare information company. The Department of Primary Care and Public Health at Imperial College is grateful for support from the National Institute for Health Research Biomedical Research Centre scheme and the National Institute for Health Research Collaboration for Leadership in Applied Health Research and Care scheme. Support was also received from the Medical Research Council and the Engineering and Physical Sciences Research Council.

Competing interests None.

Ethics approval Section 60 approval was obtained from the Patient Information Advisory Group (PIAG) to hold confidential data and analyse them for research purposes. Consent was given on behalf of patients since, for national data, individual consent is considered unfeasible. Ethics approval was also obtained from St Mary's local research ethics committee.

Provenance and peer review Not commissioned; externally peer reviewed.

\section{REFERENCES}

1. Iwane MK, Edwards KM, Szilagyi PG, et al. Population-based surveillance for hospitalizations associated with respiratory syncytial virus, influenza virus, and parainfluenza viruses among young children. Pediatrics 2004;113:1758-64.

2. National Institute for Health and Clinical Excellence (NICE). Respiratory tract infections - antibiotic prescribing. 2008. http://www.nice.org.uk/nicemedia/pdf/ CG69FullGuideline.pdf (accessed Sep 2009).

3. Gulliford M, Latinovic R, Charlton J, et al. Selective decrease in consultations and antibiotic prescribing for acute respiratory tract infections in UK primary care up to 2006. J Public Health 2009;31:512-20.

4. Majeed A, Williams S, Jarman B, et al. Prescribing of antibiotics and admissions for respiratory tract infections in England. BMJ 2004;329:879.

5. Mainous AGI, Saxena S, Hueston WJ, et al. Ambulatory antibiotic prescribing for acute bronchitis and cough and hospital admissions for respiratory infections: time trends analysis. J R Soc Med 2006:99:358-62.

6. Price DB, Honeybourne D, Little $\mathrm{P}$, et al. Community-acquired pneumonia mortality: a potential link to antibiotic prescribing trends in general practice. Respir Med 2004:98:17-24.

7. Eastham KM, Freeman R, Kearns AM, et al. Clinical features, aetiology and outcome of empyema in children in the north east of England. Thorax 2004;59:522-5.

8. Gupta R, Crowley S. Increasing paediatric empyema admissions. Thorax 2006;61:179-80.

9. Playfor SD, Stewart RJ, Smith CM, et al. Childhood empyema. Thorax 1999;54:92.

10. Roxburgh CS, Youngson GG, Townend JA, et al. Trends in pneumonia and empyema in Scottish children in the past 25 years. Arch Dis Child 2008;93:316-18.

11. Hendrickson DJ, Blumberg DA, Joad JP, et al. Five-fold increase in pediatric parapneumonic empyema since introduction of pneumococcal conjugate vaccine. Pediatr Infect Dis J 2008;27:1030-2.
12. Obando I, Munoz-Almagro C, Arroyo LA, et al. Pediatric parapneumonic empyema, Spain. Emerg Infect Dis 2008;14:1390-7.

13. Goldbart AD, Leibovitz E, Porat N, et al. Complicated community acquired pneumonia in children prior to the introduction of the pneumococcal conjugated vaccine. Scand J Infect Dis 2009;41:182-7.

14. Tan T0, Mason EO Jr, Wald ER, et al. Clinical characteristics of children with complicated pneumonia caused by Streptococcus pneumoniae. Pediatrics 2002;110:1-6

15. Trotter CL, Stuart JM, George R, et al. Increasing hospital admissions for pneumonia, England. Emerg Infect Dis 2008;14:727-33.

16. Grijalva CG, Nuorti JP, Arbogast PG, et al. Decline in pneumonia admissions afte routine childhood immunisation with pneumococcal conjugate vaccine in the USA: a time-series analysis. Lancet 2007;369:1179-86.

17. Health Protection Agency. Invasive pneumococcal disease (IPD) in England and Wales after 7-valent conjugate vaccine (PCV7); potential impact of 10 and 13-valent vaccines. http://www.hpa.org.uk/web/HPAwebFile/HPAweb_C/1245581527892 (accessed Jan 2010).

18. Eltringham G, Kearns A, Freeman R, et al. Culture-negative childhood empyema is usually due to penicillin-sensitive Streptococcus pneumoniae capsular serotype 1. J Clin Microbiol 2003;41:521-2.

19. Byington CL, Korgenski K, Daly J, et al. Impact of the pneumococcal conjugate vaccine on pneumococcal parapneumonic empyema. Pediatr Infect Dis $\mathrm{J}$ 2006:25:250-4.

20. Health Protection Agency. Invasive pneumococcal disease (IPD) in England and Wales after 7-valent conjugate vaccine (PCV7). 2009. http://www.hpa.org.uk/web/ HPAweb\&HPAwebStandard/HPAweb C/1207821646480 (accessed Jan 2010)

21. World Health Organisation (WHO). International Classification of Diseases (ICD) Version 10. 2009. http://apps.who.int/classifications/apps/icd/icd10online/ laccessed Sep 2009).

22. Bottle A, Aylin P, Majeed A. Identifying patients at high risk of emergency hospital admissions: a logistic regression analysis. J $R$ Soc Med 2006:99:406-14.

23. The Audit Commission. Data remember: improving the quality of patient-based information in the NHS. London: The Audit Commission, 2002.

24. Saxena S, Bottle A, Gilbert R, et al. Increasing short-stay unplanned hospital admissions among children in England; time trends analysis '97-'06. PLoS One 2009; 4:e7484.

25. Thompson PL, Spyridis N, Sharland M, et al. Changes in clinical indications for community antibiotic prescribing for children in the UK from 1996 to 2006: will the new NICE prescribing guidance on upper respiratory tract infections just be ignored? Arch Dis Child 2009;94:337-40.

26. Winchester CC, Macfarlane T, Thomas M, et al. Antibiotic prescribing and outcomes of lower respiratory tract infection in UK primary care. Chest 2009;135:1163-72.

27. Saglani S, Harris KA, Wallis $C$, et al. Empyema: the use of broad range $16 \mathrm{~S}$ rDNA PCR for pathogen detection. Arch Dis Child 2005:90:70-3.

28. Fletcher M, Leeming J, Cartwright $\mathrm{K}$, et al. Childhood empyema: limited potentia impact of 7-valent pneumococcal conjugate vaccine. Pediatr Infect Dis J 2006;25:559-60.

29. Pilishvili T, Lexau C, Farley MM, et al. Sustained reductions in invasive pneumococcal disease in the era of conjugate vaccine. $J$ Infect Dis 2010;201:32-41

30. Whitney CG, Farley MM, Hadler J, et al. Decline in invasive pneumococcal disease after the introduction of protein-polysaccharide conjugate vaccine. $N$ Engl J Med 2003;348:1737-46

31. Li ST, Tancredi DJ. Empyema hospitalizations increased in US children despite pneumococcal conjugate vaccine. Pediatrics 2010:125:26-33

32. Park SY, Van Beneden CA, Pilishvili T, et al. Invasive pneumococcal infections among vaccinated children in the United States. J Pediatr 2009:156:478-83.e2.

33. Centres for Disease Control and Prevention. Vaccines and immunizations. Atlanta, Georgia: Department of Health and Human Services, CDC, 2010. http://www.cdc.gov/vaccines/.

34. Meropol SB, Chen Z, Metlay JP. Reduced antibiotic prescribing for acute respiratory infections in adults and children. Br J Gen Pract 2009;59:e321-8. 